\title{
Finite Element Multigrid Method for the Boundary Value Problem of Fractional Advection Dispersion Equation
}

\author{
Zhiqiang Zhou and Hongying Wu \\ Department of Mathematics, Huaihua University, Huaihua 418008, China \\ Correspondence should be addressed to Zhiqiang Zhou; zhiqzhou@263.net
}

Received 2 March 2013; Accepted 14 May 2013

Academic Editor: Morteza Rafei

Copyright (c) 2013 Z. Zhou and H. Wu. This is an open access article distributed under the Creative Commons Attribution License, which permits unrestricted use, distribution, and reproduction in any medium, provided the original work is properly cited.

\begin{abstract}
The stationary fractional advection dispersion equation is discretized by linear finite element scheme, and a full V-cycle multigrid method (FV-MGM) is proposed to solve the resulting system. Some useful properties of the approximation and smoothing operators are proved. Using these properties we derive the convergence results in both $L^{2}$ norm and energy norm for FV-MGM. Numerical examples are given to demonstrate the convergence rate and efficiency of the method.
\end{abstract}

\section{Introduction}

We investigate the finite element full V-cycle multigrid method (FV-MGM) to the boundary value problem of linear stationary fractional advection dispersion equation (FADE).

Problem 1. Given $\Omega=(0,1), f: \bar{\Omega} \rightarrow \mathbb{R}$, find $u: \bar{\Omega} \rightarrow \mathbb{R}$ such that

$$
\begin{gathered}
-\frac{1}{2} D\left({ }_{0} D_{x}^{-\beta}+{ }_{x} D_{1}^{-\beta}\right) D u+c(x) u=f(x), \quad \text { in } \Omega, \\
u=0, \quad \text { on } \partial \Omega,
\end{gathered}
$$

where $0<\beta<1, c(x) \geq 0, D$ represents a single spatial derivative, and ${ }_{0} D_{x}^{-\beta}$ and ${ }_{x} D_{1}^{-\beta}$ represent Riemann-Liouville left and right fractional integral operators, respectively [1-3].

FADE has been used in modeling physical phenomena exhibiting anomalous diffusion, that is, diffusion not accurately modeled by the usual advection dispersion equation [4-7]. For example, solutes moving through aquifers do not generally follow a Fickian, second-order, and governing equation because of large deviations from the stochastic process of Brownian motion [8-10]. Many scholars developed numerical methods, including finite difference method [11], finite element method [12-14], spectral method [15] and moving collocation method [16] to solve FADEs. Most of them used Gauss elimination method or conjugate gradient norm residual method to solve the resulting system, so the computational complexity is $\mathcal{O}\left(N^{3}\right)$ or $\mathcal{O}\left(N \log ^{2} N\right)$. To date only a few of them considered MGM numerical methods. For example, Pang and Sun [11] developed an MGM to solve the linear system with Toeplitz-like structure, but the fractional derivatives are defined in the Grünwald-Letnikov form and the discretized system is obtained by difference scheme. It motivates us to design a fast MGM algorithm to deal with FE equations of FADE.

In this paper, we follow the ideas in $[17,18]$ to develop a FV-MGM for solving the resulting system of Problem 1 discretized by linear finite element method. By selecting appropriate iteration operator and iteration numbers, we prove that FV-MGM has the same convergence rate as classic FEM and the computational cost increases linearly with respect to the increasing of unknown variables.

The remaining of this paper is organized as follows. The next section recalls the variational formulation of the above FADE and corresponding convergence results. In Section 3 we describe the multigrid algorithm, estimate the spectral radius of FE equations, show the properties of approximation and soothing operators, and prove the convergence theorems for FV-MGM. Numerical examples demonstrating convergence rate and computational work are presented in Section 4. Concluding remarks are given in the final section. 


\section{Variational Formulation and Convergence Results}

Let $\alpha:=(2-\beta) / 2$, so that $1 / 2<\alpha \leq 1$. Define the associate bilinear form $B: H_{0}^{\alpha}(\Omega) \times H_{0}^{\alpha}(\Omega) \rightarrow \mathbb{R}$ as

$$
\begin{aligned}
B(u, v):= & \frac{1}{2}\left\langle{ }_{0} D_{x}^{-\beta} D u, D v\right\rangle \\
& +\frac{1}{2}\left\langle{ }_{x} D_{1}^{-\beta} D u, D v\right\rangle+(c(x) u, v),
\end{aligned}
$$

and linear functional $F: H_{0}^{\alpha}(\Omega) \rightarrow \mathbb{R}$ as

$$
F(v):=\langle f, v\rangle
$$

where $(\cdot, \cdot)$ denotes the inner product on $L^{2}(\Omega)$ and $\langle\cdot, \cdot\rangle$ the duality pairing of $H^{-\mu}(\Omega)$ and $H_{0}^{\mu}(\Omega), \mu \geq 0$.

Thus, the Galerkin variational solution of Problem 1 may be defined as follows.

Definition 2 (variational solution). A function $u \in H_{0}^{\alpha}(\Omega)$ is a variational solution of Problem 1 provided that

$$
B(u, v)=F(v), \quad \forall v \in H_{0}^{\alpha}(\Omega) .
$$

Ervin and Roop ([17], 2006) proved that the bilinear form $B(\cdot, \cdot)$ satisfies the coercivity and continuity; that is, there exist two positive constants $C_{1}$ and $C_{2}$ such that

$$
\begin{gathered}
B(u, u) \geq C_{1}\|u\|_{H^{\alpha}(\Omega)}^{2}, \quad \forall u \in H_{0}^{\alpha}(\Omega), \\
B(u, v) \leq C_{2}\|u\|_{H^{\alpha}(\Omega)}\|v\|_{H^{\alpha}(\Omega)}, \quad \forall u, v \in H_{0}^{\alpha}(\Omega) .
\end{gathered}
$$

Hence, they claimed that there exists a unique solution to (4). Note that from (5) and (6) we have norm equivalence of $\|\cdot\|_{H^{\alpha}(\Omega)}$ and energy norm $\|\cdot\|_{E}=B(\cdot, \cdot)^{1 / 2}$, that is,

$$
\sqrt{C_{1}}\|v\|_{H^{\alpha}(\Omega)} \leq\|v\|_{E} \leq \sqrt{C_{2}}\|v\|_{H^{\alpha}(\Omega)}, \quad \forall v \in H_{0}^{\alpha}(\Omega) .
$$

Let $S_{h}$ denote a partition of $\Omega$ such that $\bar{\Omega}=\{\bigcup K: K \in$ $\left.S_{h}\right\}$. Assume that there exists a positive constant $\rho$ such that $\rho h_{k} \leq h_{K} \leq h_{k}$, where $h_{k}=\max _{K \in S_{h}} h_{K}$. Let $P_{m}(K)$ denote the space of polynomials of degree less than or equal to $m$ on $K \in S_{h}$. Associated with $S_{h}$, define the finite-dimensional subspace $X_{h} \subset H_{0}^{\alpha}(\Omega)$ as

$$
X_{h}:=\left\{v \in H_{0}^{\alpha}(\Omega) \cap C^{0}(\bar{\Omega}):\left.v\right|_{K} \in P_{m-1}(K), \forall K \in S_{h}\right\} .
$$

Let $u_{h}$ be the solution to the finite-dimensional variational problem:

$$
B\left(u_{h}, v_{h}\right)=F\left(v_{h}\right), \quad \forall v_{h} \in X_{h} .
$$

Note that the existence and uniqueness of solution to (9) follow from the fact that $X_{h}$ is a subset of the space $H_{0}^{\alpha}(\Omega) \cap H_{0}^{m}(\Omega)$. Ervin and Roop have obtained convergence estimate in the energy norm $\|\cdot\|_{E}$. At the same time, under the assumption concerning the regularity of the solution to the adjoint problem of Problem 1 [17, Assumption 4.1], convergence estimate in the $L^{2}$ norm was proved.
Theorem 3 (see [17, Corollary 4.3]). Let $u \in H_{0}^{\alpha}(\Omega) \bigcap$ $H^{r}(\Omega)(\alpha \leq r \leq m)$ solve (4) and $u_{h}$ solve (9). Then there exists a constant $C_{3}>0$ such that $e=u-u_{h}$ satisfies

$$
\|e\|_{H^{\alpha}(\Omega)} \leq C_{3} h_{k}^{r-\alpha}\|u\|_{H^{r}(\Omega)} .
$$

Theorem 4 (see [17, Theorem 4.4]). Let $u \in H_{0}^{\alpha}(\Omega) \bigcap$ $H^{r}(\Omega)(\alpha \leq r \leq m)$ solve $(4)$ and $u_{h}$ solve $(9)$, where $m-1$ is the degree of Galerkin finite element model. Then, if the regularity of the solution to the adjoint problem is satisfied, there exists a constant $C_{4}\left(\right.$ or $\left.C_{4}^{\prime}\right)$ such that the error $e=u-u_{h}$ satisfies

$$
\begin{gathered}
\|e\|_{L^{2}(\Omega)} \leq C_{4} h_{k}^{\alpha}\|u\|_{E}, \quad \alpha \neq \frac{3}{4}, \\
\|e\|_{L^{2}(\Omega)} \leq C_{4}^{\prime} h_{k}^{\alpha-\varepsilon}\|u\|_{E}, \quad \alpha=\frac{3}{4}, \quad 0<\forall \varepsilon<\frac{1}{2} .
\end{gathered}
$$

From Theorems 3 and 4 and the equivalence of $\|\cdot\|_{H^{\alpha}(\Omega)}$ and $\|\cdot\|_{E}$, we have the error estimates in the $L^{2}$ norm.

Theorem 5. Under the same assumptions in Theorem 4, one has

$$
\begin{gathered}
\|e\|_{L^{2}(\Omega)} \leq C_{5} h_{k}^{r}\|u\|_{H^{r}(\Omega)}, \quad \alpha \neq \frac{3}{4}, \\
\|e\|_{L^{2}(\Omega)} \leq C_{5} h_{k}^{r-\varepsilon}\|u\|_{H^{r}(\Omega)}, \quad \alpha=\frac{3}{4}, \quad 0<\forall \varepsilon<\frac{1}{2},
\end{gathered}
$$

where $C_{5}=\sqrt{C_{2}} C_{3} C_{4}\left(\right.$ or $\left.C_{5}=\sqrt{C_{2}} C_{3} C_{4}^{\prime}\right)$.

\section{Multigrid Method for FADE}

For the simpleness, we only discuss the case of linear finite element, and it is necessary to restrict the mesh partition. Let $\left\{\mathscr{T}_{k}\right\}$ denote a sequence of partitions of $\bar{\Omega}$ and $h_{k}$ be the mesh size of $\mathscr{T}_{k}$. From now on, we assume that $\left\{\mathscr{T}_{k}\right\}$ is a quasiuniform family, that is,

$$
\rho h_{k} \leq x_{i}-x_{i-1} \leq h_{k}, \quad i=1,2, \ldots, N_{k},
$$

with positive constant $\rho$. At the same time, let $\mathscr{T}_{k}$ be obtained from $\mathscr{T}_{k-1}$ via a regular subdivision, that is, $\mathscr{T}_{k-1} \subset \mathscr{T}_{k}$ for $k \geq 2$. Let $V_{k}$ denote $C^{0}$ piecewise linear functions with respect to $\mathscr{T}_{k}$ that vanish on $\partial \Omega$.

\subsection{Mesh-Dependent Norms}

Definition 6. The mesh-dependent inner product $(\cdot, \cdot)_{k}$ on $V_{k}$ is defined by

$$
(v, w)_{k}=h_{k} \sum_{i=1}^{N_{k}-1} v\left(x_{i}\right) w\left(x_{i}\right) .
$$

Definition 7. The operator $A_{k}: V_{k} \rightarrow V_{k}$ is defined by

$$
\left(A_{k} v, w\right)_{k}=B(v, w), \quad \forall v, w \in V_{k} .
$$

In terms of the operator $A_{k}$, the discretized equation of (9) can be written as

$$
A_{k} u_{k}=f_{k}
$$


where $f_{k} \in V_{k}$ satisfies

$$
\left(f_{k}, v_{k}\right)=(f, v), \quad \forall v \in V_{k} .
$$

Remark 8. (i) From the Riesz representation theorem, we point out that the operator $A_{k}$ is defined uniquely. (ii) Since $B(v, w)$ is symmetric and satisfies the proposition of coercivity, $A_{k}$ is symmetric positive definite woth respect to $(\cdot, \cdot)_{k}$.

The mesh-dependent norms are defined by

$$
\||v|\|_{s, k}=\sqrt{\left(A_{k}^{s} v, v\right)_{k}} .
$$

Observe that the energy norm $\|\cdot\|_{E}$ coincides with $\||\cdot|\|_{1, k}$ norm on $V_{k}$. Similarly, $\||\cdot|\|_{0, k}$ is the norm associated with the mesh-dependent inner product (14). The following lemma shows that $\||\cdot|\|_{0, k}$ is equivalent to the $L^{2}$ norm.

Lemma 9. The norm $\|\cdot\|_{L^{2}(\Omega)}$ is equivalent to mesh-depentent norm $\||\cdot|\|_{0, k}$, that is,

$$
\rho\left\|v\left|\left\|_{0, k} \leq\right\| v\left\|_{L^{2}(\Omega)} \leq\right\|\right| v \mid\right\|_{0, k}, \quad \forall v \in V_{k} .
$$

Particularly, $\||\cdot|\|_{0, k}=\|\cdot\|_{L^{2}(\Omega)}$ for the uniform mesh.

Proof. The proof is analogous to Lemma 6.2.7 in reference [18].

In order to estimate the spectral radius, $\Lambda\left(A_{k}\right)$, of $A_{k}$, we need the following norm interpolation lemma (see [19, Theorem 1.3.7].

Lemma 10 (interpolation theorem). Assume that $\Omega$ is an open set of $\mathbb{R}^{d}$ with a Lipchitz continuous boundary. Let $s_{1}<s_{2}$ be two real numbers, and $\mu=(1-\theta) s_{1}+\theta s_{2}, 0 \leq \theta \leq 1$. Then there exists a constant $C_{6}>0$ such that

$$
\|u\|_{H^{\mu}(\Omega)} \leq C_{6}\|u\|_{H^{s_{1}}(\Omega)}^{1-\theta}\|u\|_{H^{s_{2}}(\Omega)}^{\theta} .
$$

Lemma 11 (spectral radius theorem). We have the estimation for spectral radius $\Lambda\left(A_{k}\right)$ :

$$
\Lambda\left(A_{k}\right) \leq C_{7} h_{k}^{-2 \alpha},
$$

where $C_{7}$ is a positive constant independent of $k$.

Proof. Let $\phi \in V_{k}$. Applying the continuity (6) of $B(\cdot, \cdot)$, norm interpolation Lemma 10, and inverse estimation, we have

$$
\begin{aligned}
B(\phi, \phi) & \leq C_{2}\|\phi\|_{H^{\alpha}(\Omega)}^{2} \\
& \leq C_{2}\left(C_{6}\|\phi\|_{H^{0}(\Omega)}^{1-\alpha} \cdot\|\phi\|_{H^{1}(\Omega)}^{\alpha}\right)^{2} \\
& \leq C_{2}\left(C_{6}\|\phi\|_{H^{0}(\Omega)}^{1-\alpha} \cdot C_{8} h_{k}^{-\alpha}\|\phi\|_{H^{0}(\Omega)}^{\alpha}\right)^{2} \\
& =C h_{k}^{-2 \alpha}\|\phi\|_{H^{0}(\Omega)}^{2} \\
& =C h_{k}^{-2 \alpha}\|\phi\|_{L^{2}(\Omega)}^{2},
\end{aligned}
$$

where $C_{8}>0$ is the constant of inverse estimate and $C=$ $\mathrm{C}_{2} \mathrm{C}_{6}^{2} \mathrm{C}_{8}^{2}$.

Let $\lambda$ be an eigenvalue of $A_{k}$ with corresponding eigenvector $\phi \in V_{k}$. From Lemma 9, we have

$$
\begin{aligned}
B(\phi, \phi) & =\left(A_{k} \phi, \phi\right)_{k} \\
& =\lambda(\phi, \phi)_{k} \\
& =\lambda\||\phi|\|_{0, k}^{2} \\
& =\lambda\|\phi\|_{L^{2}(\Omega)}^{2} .
\end{aligned}
$$

Combining (22) and (23), the following inequality holds:

$$
\lambda=\frac{B(\phi, \phi)}{\|\phi\|_{L^{2}(\Omega)}^{2}} \leq \frac{C h_{k}^{-2 \alpha}\|\phi\|_{L^{2}(\Omega)}^{2}}{\|\phi\|_{L^{2}(\Omega)}^{2}}=C_{7} h_{k}^{-2 \alpha},
$$

where $C_{7}=C_{2} C_{6}^{2} C_{8}^{2}$.

3.2. The V-Cycle Multigrid Algorithm. Firstly, we give the intergrid transfer operators which play a very important role in convergence analysis.

Definition 12 (intergrid transfer operator). The coarse-to-fine intergrid transfer operator $I_{k-1}^{k}: V_{k-1} \rightarrow V_{k}$ is taken to be the natural injection, that is,

$$
I_{k-1}^{k} v=v, \quad \forall v \in V_{k-1} .
$$

The fine-to-coarse operator $I_{k}^{k-1}: V_{k} \rightarrow V_{k-1}$ is defined by

$$
\left(I_{k}^{k-1} w, v\right)_{k-1}=\left(w, I_{k-1}^{k} v\right)_{k}=(w, v)_{k}, \quad \forall v \in V_{k-1}, w \in V_{k} .
$$

Now, we describe the $k$ th level of V-cycle MGM (VMGM) and full V-cycle MGM. Let $m$ be the smoothing number, $n_{k}$ the iteration number of $k$ th level V-MGM, and $\Lambda_{k}$ be a parameter dependent on $k$. Denote $M G\left(k, z_{0}, g\right)$ the approximate solution of the $A_{k} z=g$ obtained from the $k$ th level iteration with initial guess $z_{0}$. The discussion of parameters $\Lambda_{k}$ and $n_{k}$ is left to the next subsection and Section 4.

Algorithm 13 (the $k$ th level V-cycle multigrid algorithm).

BEGIN:

For $k=1: M G\left(1, z_{0}, g\right)=A_{1}^{-1} g$.

For $k>1, M G\left(k, z_{0}, g\right)$ is obtained recursively in three steps.

Presmoothing Step. For $1 \leq l \leq m$, let $z_{l}=z_{l-1}+$ $\left(1 / \Lambda_{k}\right)\left(g-A_{k} z_{l-1}\right)$.

Error Correction.

$$
\begin{aligned}
& \bar{g}=I_{k}^{k-1}\left(g-A_{k} z_{m}\right), \\
& q_{1}=M G(k-1,0, \bar{g}), \\
& z_{m+1}=z_{m}+I_{k-1}^{k} q_{1} .
\end{aligned}
$$


Postsmoothing step. For $m+1 \leq l \leq 2 m+1$, let $z_{l}=z_{l-1}+\left(1 / \Lambda_{k}\right)\left(g-A_{k} z_{l-1}\right)$.

The output of the $k$ th level iteration is $M G\left(k, z_{0}, g\right):=z_{2 m+1}$.

\section{END.}

Algorithm 14 (the full V-cycle multigrid algorithm).

\section{BEGIN:}

For $k=1, \widehat{u}_{1}=A_{1}^{-1} f_{1}$.

For $k \geq 2$,

$$
\begin{aligned}
& u_{0}^{k}=I_{k-1}^{k} \widehat{u}_{k-1}, \\
& u_{l}^{k}=M G\left(k, u_{l-1}^{k}, f_{k}\right), \quad 1 \leq l \leq n_{k}, \\
& \widehat{u}_{k}=u_{n_{k}}^{k} .
\end{aligned}
$$

END.

3.3. Approximation and Smoothing Properties. In this subsection, we prove some properties of projection operator $P_{k-1}$ and smoothing operator $R_{k}$, which are the key ingredients for V-MGM and FV-MGM algorithm.

Definition 15. Let $P_{k}: V \rightarrow V_{k}$ be the orthogonal projection with respect to $B(\cdot, \cdot)$, that is,

$$
B(v, w)=B\left(P_{k} v, w\right), \quad \forall w \in V_{k} .
$$

Definition 16. Define the relaxation operator:

$$
R_{k}:=I-\frac{1}{\Lambda_{k}} A_{k}
$$

Throughout this paper, we assume that $\Lambda_{k}$ denotes some upper bound for the spectral radius of $A_{k}$ satisfying $\Lambda_{k} \leq$ $C_{7} h_{k}^{-2 \alpha}$.

Lemma 17. Let $v \in V_{k}, \bar{g}=I_{k}^{k-1} A_{k} v$, and $q \in V_{k-1}$ satisfy the equation $A_{k-1} q=\bar{g}$. Then $q=P_{k-1} v$.

Proof. For $w \in V_{k-1}$,

$$
\begin{aligned}
B(q, w) & =\left(A_{k-1} q, w\right)_{k-1} \\
& =(\bar{g}, w)_{k-1} \\
& =\left(I_{k}^{k-1} A_{k} v, w\right)_{k-1} \\
& =\left(A_{k} v, w\right)_{k} \\
& =B(v, w) .
\end{aligned}
$$

Therefore, from the definition of $P_{k-1}$ we have $q=P_{k-1} v$.

Remark 18. Let $g=A_{k} v$. From the Riesz representation theorem there exists a unique $f \in H^{-\alpha}(\Omega)$ such that

$$
(g, w)_{k}=\langle f, w\rangle, \quad \forall w \in V_{k},
$$

which means that $v$ is a finite element solution of variational problem (4) on $V_{k}$. Observing that

$$
\begin{aligned}
(\bar{g}, w)_{k-1} & =\left(I_{k}^{k-1} g, w\right)_{k-1} \\
& =(g, w)_{k}=\langle f, w\rangle, \quad \forall w \in V_{k-1},
\end{aligned}
$$

we claim that $q=P_{k-1} v$ is also a finite element solution of variational problem (4) on $V_{k-1}$. Therefore, noting $V_{k-1} \subset V_{k}$ (since $\mathscr{T}_{k-1} \subset \mathscr{T}_{k}$ ) and applying Theorem 4, we have

$$
\begin{gathered}
\left\|v-P_{k-1} v\right\|_{L^{2}(\Omega)} \leq C_{4} h_{k}^{\alpha}\left\|v-P_{k-1} v\right\|_{E}, \quad \alpha \neq \frac{3}{4}, \\
\left\|v-P_{k-1} v\right\|_{L^{2}(\Omega)} \leq C_{4}^{\prime} h_{k}^{\alpha-\varepsilon}\left\|v-P_{k-1} v\right\|_{E}, \quad \alpha=\frac{3}{4} .
\end{gathered}
$$

Lemma 19. There exists a positive constant $C_{9}$ such that

$$
\begin{aligned}
& \|\|\left(I-P_{k-1}\right) v \mid \|_{0, k} \\
& \quad \leq C_{9} h_{k}^{\alpha}\left\|\left|\left(I-P_{k-1}\right) v\right|\right\|_{1, k}, \quad \forall v \in V_{k}, \alpha \neq \frac{3}{4}, \\
& \|\|\left(I-P_{k-1}\right) v \mid \|_{0, k} \\
& \quad \leq C_{9} h_{k}^{\alpha-\varepsilon}\left\|\left(I-P_{k-1}\right) v \mid\right\|_{1, k}, \quad \forall v \in V_{k}, \alpha=\frac{3}{4}, 0<\varepsilon<\frac{1}{2} .
\end{aligned}
$$

Proof. We only prove the case $\alpha \neq 3 / 4$, and the proof for the other case is analogous. Applying Remark 18 and Lemma 9 we have

$$
\begin{aligned}
\left\|\left(I-P_{k-1}\right) v \mid\right\|_{0, k} & \leq \frac{1}{\rho}\left\|\left(I-P_{k-1}\right) v\right\|_{L^{2}(\Omega)} \\
& \leq \frac{C_{4}}{\rho} h_{k}^{\alpha}\left\|\left(v-P_{k-1} v\right)\right\|_{E} \\
& =C_{9} h_{k}^{\alpha}\left\|\left(I-P_{k-1}\right) v \mid\right\|_{1, k},
\end{aligned}
$$

where $C_{9}=C_{4} / \rho$.

Lemma 20. There exists a positive constant $C_{9}$ such that

$$
\begin{aligned}
& \left\|\left(I-P_{k-1}\right) v\left|\left\|_{1, k} \leq C_{9} h_{k}^{\alpha}\right\|\right| v \mid\right\|_{2, k}, \quad \forall v \in V_{k}, \alpha \neq \frac{3}{4}, \\
& \left\|\left(I-P_{k-1}\right) v \mid\right\|_{1, k} \\
& \quad \leq C_{9} h_{k}^{\alpha-\varepsilon}\||v|\|_{2, k}, \quad \forall v \in V_{k}, \quad \alpha=\frac{3}{4}, 0<\varepsilon<\frac{1}{2} .
\end{aligned}
$$


Proof. Applying projection operator $P_{k-1}$, generalized Cauchy-Schwarz inequality (see [18, Lemma 6.2.10]), and Lemma 19

$$
\begin{aligned}
\left\|\left(I-P_{k-1}\right) v \mid\right\|_{1, k}^{2} & =\left\|\left(I-P_{k-1}\right) v\right\|_{E}^{2} \\
& =B\left(v-P_{k-1} v, v-P_{k-1} v\right) \\
& =B\left(v-P_{k-1} v, v\right) \\
& \leq\left\|\left(I-P_{k-1}\right) v\left|\left\|_{0, k}\right\|\right| v \mid\right\|_{2, k} \\
& \leq C_{9} h_{k}^{\alpha}\left\|\left|\left(I-P_{k-1}\right) v\right|\right\|_{1, k}\||v|\|_{2, k} \\
& =C_{9} h_{k}^{\alpha}\left\|\left(I-P_{k-1}\right) v\left|\left\|_{1, k}\right\|\right| v \mid\right\|_{2, k}
\end{aligned}
$$

if $\alpha \neq 3 / 4$. Therefore, dividing through by $\left\|\left|\left(I-P_{k-1}\right) v\right|\right\|_{1, k}$ yields (35). The case $\alpha=3 / 4$ is analogous.

Lemma 21. Let $m$ be the number of smoothing steps. Then

$$
B\left(\left(I-R_{k}\right) R_{k}^{2 m} v, v\right) \leq \frac{1}{2 m} B\left(\left(I-R_{k}^{2 m}\right) v, v\right) .
$$

Proof. See Lemma 6.6.7 in [18].

Lemma 22. Let $m$ be the number of smoothing steps. Then there exists a positive constant $C_{10}$ such that

$$
B\left(\left(I-P_{k-1}\right) R_{k}^{m} v,\left(I-P_{k-1}\right) R_{k}^{m} v\right) \leq \frac{C_{10}}{m} B\left(\left(I-R_{k}^{2 m}\right) v, v\right) .
$$

Proof. Applying Lemmas 20, 11, and 21, we have

$$
\begin{aligned}
B & \left(\left(I-P_{k-1}\right) R_{k}^{m} v,\left(I-P_{k-1}\right) R_{k}^{m} v\right) \\
& =\left\|\left(I-P_{k-1}\right) R_{k}^{m} v\right\|_{E}^{2} \\
& =\left\|\left|\left(I-P_{k-1}\right) R_{k}^{m} v\right|\right\|_{1, k}^{2} \\
& \leq C_{9}^{2} h_{k}^{2 \alpha}\left\|\left|R_{k}^{m} v\right|\right\|_{2, k}^{2} \\
& =C_{9}^{2} h_{k}^{2 \alpha}\left(A_{k}^{2} R_{k}^{m} v, R_{k}^{m} v\right)_{k} \\
& =C_{9}^{2} h_{k}^{2 \alpha} B\left(A_{k} R_{k}^{m} v, R_{k}^{m} v\right) \\
& =C_{9}^{2} h_{k}^{2 \alpha} \Lambda_{k} B\left(\left(I-R_{k}\right) R_{k}^{m} v, R_{k}^{m} v\right) \\
& \leq C_{9}^{2} C_{7} B\left(\left(I-R_{k}\right) R_{k}^{m} v, R_{k}^{m} v\right) \\
& =C_{9}^{2} C_{7} B\left(\left(I-R_{k}\right) R_{k}^{2 m} v, v\right) \\
& \leq \frac{C_{9}^{2} C_{7}}{(2 m) B\left(\left(I-R_{k}^{2 m}\right) v, v\right)} \\
& =\frac{C_{10}}{m B\left(\left(I-R_{k}^{2 m}\right) v, v\right)}
\end{aligned}
$$

if $\alpha \neq 3 / 4$, where $C_{10}=C_{9}^{2} C_{7} / 2$. Note that (39) also holds for $\alpha=3 / 4$ since $\varepsilon>0$.
3.4. Convergence Analysis. In this subsection, we prove convergence theorems for $\mathrm{V}$-cycle FE multigrid method.

Definition 23. The error operator of V-cycle multigrid $E_{k}$ : $V_{k} \rightarrow V_{k}$ is defined recursively by

$$
\begin{gathered}
E_{1}=0, \\
E_{k}=R_{k}^{m}\left[I-\left(I-E_{k-1}\right) P_{k-1}\right] R_{k}^{m}, \quad k>1 .
\end{gathered}
$$

Proposition 24. The operator $E_{k}$ has the following propositions (see [18, Lemma 6.6.2 and Proposition 6.6.9]):

(i) if $z, g \in V_{k}$ satisfy $A_{k} z=g$, then

$$
E_{k}\left(z-z_{0}\right)=z-M G\left(k, z_{0}, g\right),
$$

(ii) $E_{k}$ is symmetric positive semidefinite with respect to $B(\cdot, \cdot)$ for $k \geq 1$, that is,

$$
\begin{gathered}
B\left(E_{k} v, w\right)=B\left(v, E_{k} w\right), \quad \forall v, w \in V_{k}, \\
B\left(E_{k} v, v\right) \geq 0 .
\end{gathered}
$$

Theorem 25. Let $m$ be the number of smoothing steps. Then

$$
B\left(E_{k} v, v\right) \leq \frac{C_{10}}{m+C_{10}} B(v, v), \quad \forall v \in V_{k} .
$$

Proof. The proof is by induction. For $k=1$, (44) is trivially true since $E_{k}=0$. Assume that (44) holds for $k-1$. Let $\gamma=$ $C_{10} /\left(m+C_{10}\right)$; therefore $1-\gamma=\gamma m / C_{10}$. Applying induction hypothesis, Proposition 24, and Lemma 22, we have

$$
\begin{aligned}
B\left(E_{k} v, v\right)= & B\left(R_{k}^{m} v, R_{k}^{m} v\right)-B\left(P_{k-1} R_{k}^{m} v, P_{k-1} R_{k}^{m} v\right) \\
& +B\left(E_{k-1} P_{k-1} R_{k}^{m} v, P_{k-1} R_{k}^{m} v\right) \\
\leq & B\left(\left(I-P_{k-1}\right) R_{k}^{m} v,\left(I-P_{k-1}\right) R_{k}^{m} v\right) \\
& +\gamma B\left(P_{k-1} R_{k}^{m} v, P_{k-1} R_{k}^{m} v\right) \\
= & (1-\gamma) B\left(\left(I-P_{k-1}\right) R_{k}^{m} v,\left(I-P_{k-1}\right) R_{k}^{m} v\right) \\
& +\gamma B\left(\left(I-P_{k-1}\right) R_{k}^{m} v,\left(I-P_{k-1}\right) R_{k}^{m} v\right) \\
& +\gamma B\left(P_{k-1} R_{k}^{m} v, P_{k-1} R_{k}^{m} v\right) \\
\leq & \frac{(1-\gamma) C_{10}}{m B\left(\left(I-R_{k}^{2 m}\right) v, v\right)}+\gamma B\left(R_{k}^{m} v, R_{k}^{m} v\right) \\
= & \gamma B\left(\left(I-R_{k}^{2 m}\right) v, v\right)+\gamma B\left(R_{k}^{m} v, R_{k}^{m} v\right) \\
= & \gamma B(v, v),
\end{aligned}
$$

which ends the proof.

Corollary 26. Let $m$ be the number of smoothing steps. Then one has the estimation for spectral radius $\Lambda\left(E_{k}\right)$ :

$$
\Lambda\left(E_{k}\right) \leq \frac{C_{10}}{m+C_{10}} .
$$


Theorem 27 (convergence of the $k$ th level iteration for $\mathrm{V}$-cycle). Let $m$ be the number of smoothing steps. Then

$$
\left\|z-M G\left(k, z_{0}, g\right)\right\|_{E} \leq \frac{C_{10}}{m+C_{10}}\left\|z-z_{0}\right\|_{E} .
$$

Hence, the kth level iteration for any $m$ is a contraction with the contraction number independent of $k$.

Proof. From (42) in Proposition 24, it suffices to show

$$
\left\|E_{k} v\right\|_{E} \leq \frac{C_{10}}{m+C_{10}}\|v\|_{E}
$$

which can be proved by Corollary 26 .

Theorem 28 (full multigrid convergence). Let $u \in H_{0}^{\alpha}(\Omega) \bigcap$ $H^{r}(\Omega)(\alpha \leq r \leq 2)$ solve (4). If the iteration numbers in full multigrid algorithm $n_{k}$ are large enough, there exists a positive constant $C_{11}$ such that

$$
\left\|u_{k}-\widehat{u}_{k}\right\|_{E} \leq C_{11} h_{k}^{r-\alpha}\|u\|_{H^{r}(\Omega)} .
$$

Proof. For the simpleness we assume that $n_{k} \geq n \geq 1$. Define $\widehat{e}_{k}=u_{k}-\widehat{u}_{k}$. In particular, $\widehat{e}_{1}=0$. Let $\gamma=C_{10} /\left(m+C_{10}\right)\left(C_{10}\right.$ is the constant in Theorem 27). We have

$$
\begin{aligned}
&\left\|\widehat{e}_{k}\right\|_{E} \leq \gamma^{n}\left\|u_{k}-\widehat{u}_{k-1}\right\|_{E} \\
& \leq \gamma^{n}\left(\left\|u_{k}-u\right\|_{E}+\left\|u-u_{k-1}\right\|_{E}+\left\|u_{k-1}-\widehat{u}_{k-1}\right\|_{E}\right) \\
& \leq \gamma^{n}\left[\sqrt{C_{2}} C_{3} h_{k}^{r-\alpha}\|u\|_{H^{r}(\Omega)}\right. \\
&\left.\quad+\sqrt{C_{2}} C_{3} h_{k-1}^{r-\alpha}\|u\|_{H^{r}(\Omega)}+\left\|\widehat{e}_{k-1}\right\|_{E}\right] \\
&=\gamma^{n}\left[3 \sqrt{C_{2}} C_{3} h_{k}^{r-\alpha}\|u\|_{H^{r}(\Omega)}+\left\|\widehat{e}_{k-1}\right\|_{E}\right] .
\end{aligned}
$$

In the following analysis, we assume that $2 \gamma^{n}<1$ (therefore $\left.n>\log _{\gamma}^{1 / 2}\right)$. By iterating the above inequality and from continuity (6) and Theorem 3, we have

$$
\begin{aligned}
\left\|\widehat{e}_{k}\right\|_{E} \leq & 3 \sqrt{C_{2}} C_{3} \gamma^{n} h_{k}^{r-\alpha}\|u\|_{H^{r}(\Omega)} \\
& +3 \sqrt{C_{2}} C_{3} \gamma^{2 n} h_{k-1}^{r-\alpha}\|u\|_{H^{r}(\Omega)}+\cdots \\
& +3 \sqrt{C_{2}} C_{3} \gamma^{k n} h_{1}^{r-\alpha}\|u\|_{H^{r}(\Omega)} \\
\leq & 3 \sqrt{C_{2}} C_{3} \frac{\gamma^{n}}{1-2 \gamma^{n}} h_{k}^{r-\alpha}\|u\|_{H^{r}(\Omega)} \\
= & C_{11} h_{k}^{r-\alpha}\|u\|_{H^{r}(\Omega)},
\end{aligned}
$$

where $C_{11}=3 \sqrt{C_{2}} C_{3} \gamma^{n} /\left(1-2 \gamma^{n}\right)$.

The following Corollary is a natural conclusion of Theorems 5 and 28.
Corollary 29. Under the assumptions in Theorem 28, the convergence rate of multigrid solution $\widehat{u}_{k}$ in $L^{2}$ norm is $r$; that is, there exists a constant $C_{12}>0$ such that

$$
\begin{gathered}
\left\|\widehat{u}_{k}-u\right\|_{L^{2}(\Omega)} \leq C_{12} h_{k}^{r}\|u\|_{H^{r}(\Omega)}, \quad \alpha \neq \frac{3}{4}, \\
\left\|\widehat{u}_{k}-u\right\|_{L^{2}(\Omega)} \leq C_{12} h_{k}^{r-\varepsilon}\|u\|_{H^{r}(\Omega)}, \quad \alpha=\frac{3}{4}, \quad 0<\forall \varepsilon<\frac{1}{2} .
\end{gathered}
$$

We end this section with a proposition that the work involved in the full multigrid algorithm is $\mathcal{O}\left(d_{k}\right)$, where $d_{k}=$ $N_{k}-2=\operatorname{dim} V_{k}$ (see [18, Proposition 6.7.4]).

\section{Numerical Examples}

In this section we employ the FV-MGM listed as Algorithm 14 in Section 3 to solve FADEs, which demonstrate the convergence rate and involved work in Theorem 28 and its Corollary 29. The parameter $m$ is taken $5, \Lambda_{k}=C_{7} h_{k}^{-2 \alpha}$, and $n_{k}$ is controlled by the stopping criterion:

$$
\left\|A_{k} \widehat{u}_{n_{k}}^{k}-g\right\|_{L^{2}(\Omega)} \leq \mathcal{O}\left(h_{k}^{\alpha}\right) .
$$

We note that the positive constants $C_{7}$ can be estimated by testing examples on the 1 th and 2 th level meshes, that is,

$$
C_{7}=\frac{1}{2}\left(h_{1}^{2 \alpha} \Lambda\left(A_{1}\right)+h_{2}^{2 \alpha} \Lambda\left(A_{2}\right)\right) .
$$

Here we calculate $C_{7}=0.05$, and, from experimental experience, let the right side of stopping criterion be $0.085 h_{k}^{\alpha}$. All numerical experiments are run in MATLAB 7.0.0.19920(R14) on a PC with the configuration: Intel(R)Core(TM)i3-2100 CPU@3.2 GHz and 1.88 GB RAM.

Example 30. Let $c(x)=1$. It can be verified that $u(x)=x^{2}-x^{3}$ is the exact solution to the boundary value problem:

$$
\begin{gathered}
-\frac{1}{2} D\left({ }_{0} D_{x}^{-\beta}+{ }_{x} D_{1}^{-\beta}\right) D u+u=f, \\
u(0)=0, \quad u(1)=0,
\end{gathered}
$$

where

$$
\begin{gathered}
f(x)=x^{2}-x^{3}+\frac{1}{2} f_{1}(x)+\frac{1}{2} f_{2}(x), \\
f_{1}(x)=-\frac{2 x^{\beta}}{\Gamma(1+\beta)}+\frac{6 x^{1+\beta}}{\Gamma(2+\beta)}, \\
f_{2}(x)=-\frac{\beta(1-x)^{\beta-1}}{\Gamma(1+\beta)}+\frac{4(\beta+1)(1-x)^{\beta}}{\Gamma(2+\beta)} \\
-\frac{6(\beta+2)(1-x)^{\beta+1}}{\Gamma(3+\beta)} .
\end{gathered}
$$

As $u \in H_{0}^{2}(\Omega)$, Corollary 29 predicts a rate of convergence of 2 in $L^{2}$ norm. Table 1 includes numerical results over 
TABLE 1: Experimental error $\left\|u-\widehat{u}_{k}\right\|_{L^{2}(\Omega)}$ for different $\beta$.

\begin{tabular}{lccccrr}
\hline$h_{k}$ & $\beta=0.3$ & & $\beta=0.6$ & & \multicolumn{2}{c}{$\beta=0.9$} \\
& Error & Conv. rate & Error & Conv. rate & Error & $7.6044 e-3$ \\
$1 / 4$ & $9.3587 e-3$ & & $8.3460 e-3$ & & $2.6348 e-3$ \\
$1 / 8$ & $2.1751 e-3$ & 2.1388 & $1.8444 e-3$ & 2.1779 & $3.6349 e-4$ \\
$1 / 16$ & $5.0679 e-4$ & 2.1016 & $4.1362 e-4$ & 2.1568 & $2.3872 e-5$ \\
$1 / 32$ & $1.1967 e-4$ & 2.0823 & $9.4937 e-5$ & 2.1233 & 2.1692 \\
$1 / 64$ & $2.9496 e-5$ & 2.0205 & $2.2629 e-5$ & 2.0688 & $2.0343 e-5$ \\
$1 / 128$ & $7.0818 e-6$ & 2.0583 & $5.4727 e-6$ & 2.0478 & $5.0852 e-6$ & 2.0436 \\
\hline
\end{tabular}

TABLE 2: Comparisons for solving Example 30 with $\beta=0.7$ by the GE method, the CGNR method, and the FV-MGM, respectively.

\begin{tabular}{lccccccrrr}
\hline$h_{k}$ & FVMGM & \multicolumn{2}{c}{ GE } & \multicolumn{3}{c}{ CGNR } & \multicolumn{2}{c}{ FVMGM } \\
& Error & CPU(s) & $R_{\text {cpu }}$ & Iter & CPU(s) & $R_{\text {cpu }}$ & Iter $=n_{k}$ & CPU(s) & $R_{\text {cpu }}$ \\
\hline $1 / 128$ & $2.0270 e-5$ & 0.0313 & & 29 & 0.0000 & & 4 & 0.0000 \\
$1 / 256$ & $1.3811 e-5$ & 0.0625 & 0.995 & 46 & 0.0156 & & 5 & 0.0156 \\
$1 / 512$ & $1.1507 e-5$ & 0.1719 & 1.451 & 72 & 0.0313 & 0.999 & 5 & 0.0469 & 1.579 \\
$1 / 1024$ & $1.1101 e-5$ & 0.5156 & 1.580 & 112 & 0.2187 & 2.796 & 3 & 0.1250 \\
$1 / 2048$ & $1.1002 e-5$ & 2.5000 & 2.274 & 174 & 1.5625 & 2.833 & 1 & 0.2688 \\
$1 / 4096$ & $1.0849 e-5$ & 15.484 & 2.627 & 270 & 9.2031 & 2.554 & 1.103 & 0.5719 \\
\hline
\end{tabular}

a uniform partition of $[0,1]$, which support the predicted rates of convergence for different values of $\beta$.

As comparisons, we also carry out the Gauss elimination (GE) and conjugate gradient normal residual (CGNR) method with the same stopping criterion of the FV-MGM, that is,

$$
\left\|A_{k} \widehat{u}_{n_{k}}^{k}-g\right\|_{L^{2}(\Omega)} \leq 10^{-n}
$$

to solve the corresponding system. For escaping "out of memory," we define that the data type of $A_{k}$ is short float in our program. Table 2 includes CPU time (without including stiffness matrix calculating time) and iteration numbers for each of the numerical methods with $n=4$. The rate of the increasing CPU time is defined by

$$
\begin{aligned}
& R_{\mathrm{cpu}} \\
& =\frac{\log [\mathrm{CPU} \text { time on finer mesh/CPU time on coarser mesh }]}{\log [\text { dim of finer mesh/ dim of coarser mesh }]} .
\end{aligned}
$$

From the table, we see that the numbers of iterations by our FV-MGM are independent of $h_{k}$. In contrast, the CGNR method (with the initial value $z_{0}=0$ ) needs more iterations when $h_{k}$ decrease. Similarly, the CPU time needed for GE and CGNR method increases much faster than that of the FVMGM.

\section{Concluding Remarks}

In general the discretized system of FADE has a full and ill-conditioned coefficient matrix, so FV-MGM is a highefficient algorithm for solving these equations. Theorems and examples in this paper show that the convergence rate of $\mathrm{FV}$ MGM is the same as classic FEM under the stopping criterion (53), and the computational work is only $\mathcal{O}\left(\operatorname{dim} V_{k}\right)$ while the stopping criterion is taken (57). Different from integerorder equations, all the convergence analysis is on fractional
Sobolev spaces $H^{\alpha}(\Omega)$. The nonsymmetric form of FADE will be studied in the future.

\section{References}

[1] I. Podlubny, Fractional Differential Equations, Academic Press, New York, NY, USA, 1999.

[2] K. B. Oldham and J. Spanier, The Fractional Calculus, Academic Press, New York, NY, USA, 1974.

[3] K. S. Miller and B. Ross, An Introduction to the Fractional Calculus and Fractional Differential Equations, John Wiley \& Sons, New York, NY, USA, 1993.

[4] B. A. Carreras, V. E. Lynch, and G. M. Zaslavsky, "Anomalous diffusion and exit time distribution of particle tracers in plasma turbulence models," Physics of Plasmas, vol. 8, no. 12, pp. 50965103, 2001.

[5] M. F. Shlesinger, B. J. West, and J. Klafter, "Lévy dynamics of enhanced diffusion: application to turbulence," Physical Review Letters, vol. 58, no. 11, pp. 1100-1103, 1987. 
[6] G. M. Zaslavsky, D. Stevens, and H. Weitzner, "Self-similar transport in incomplete chaos," Physical Review E, vol. 48, no. 3, pp. 1683-1694, 1993.

[7] F. Mainardi, "Fractional calculus: some basic problems in continuum and statistical mechanics," in Fractals and Fractional Calculus in Continuum Mechanics, pp. 291-348, Springer, New York, NY, USA, 1997.

[8] D. A. Benson, S. W. Wheatcraft, and M. M. Meerschaeert, "The fractional-order governing equation of Lévy motion," Water Resources Research, vol. 36, no. 6, pp. 1403-1412, 2000.

[9] D. A. Benson, S. W. Wheatcraft, and M. M. Meerschaeert, "Application of fractional advection-dispersion equation," Water Resources Research, vol. 36, no. 6, pp. 1413-1423, 2000.

[10] D. A. Benson, R. Schumer, M. M. Meerschaert, and S. W. Wheatcraft, "Fractional dispersion, Lévy motion, and the MADE tracer tests," Transport in Porous Media, vol. 42, no. 12, pp. 211-240, 2001.

[11] H.-K. Pang and H.-W. Sun, "Multigrid method for fractional diffusion equations," Journal of Computational Physics, vol. 231, no. 2, pp. 693-703, 2012.

[12] V. J. Ervin and J. P. Roop, "Variational solution of fractional advection dispersion equations on bounded domains in $\mathbb{R}^{d,}$ Numerical Methods for Partial Differential Equations, vol. 23, no. 2, pp. 256-281, 2007.

[13] W. H. Deng, "Finite element method for the space and time fractional Fokker-Planck equation," SIAM Journal on Numerical Analysis, vol. 47, no. 1, pp. 204-226, 2008.

[14] C. M. Chen and T. Shih, Finite Element Methods for Integrodifferential Equations, World Scientific, Singapore, 1998.

[15] X. J. Li and C. J. Xu, "Existence and uniqueness of the weak solution of the space-time fractional diffusion equation and a spectral method approximation," Communications in Computational Physics, vol. 8, no. 5, pp. 1016-1051, 2010.

[16] J. T. Ma and Y. J. Jiang, "Moving collocation methods for time fractional differential equations and simulation of blowup," Science China Mathematics, vol. 54, no. 3, pp. 611-622, 2011.

[17] V. J. Ervin and J. P. Roop, "Variational formulation for the stationary fractional advection dispersion equation," Numerical Methods for Partial Differential Equations, vol. 22, no. 3, pp. 558576, 2006.

[18] S. C. Brenner and L. R. Scott, The Mathematical Theory of Finite Element Methods, Springer, New York, NY, USA, 1994.

[19] A. Quarteroni and A. Valli, Numerical Approximation of Partial Differential Equations, Springer, Berlin, Germany, 1994. 


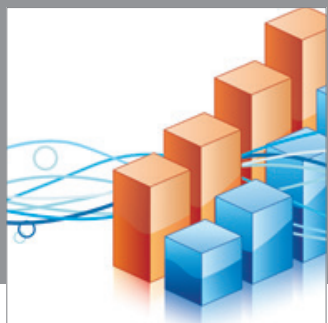

Advances in

Operations Research

mansans

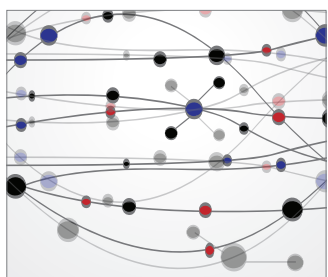

The Scientific World Journal
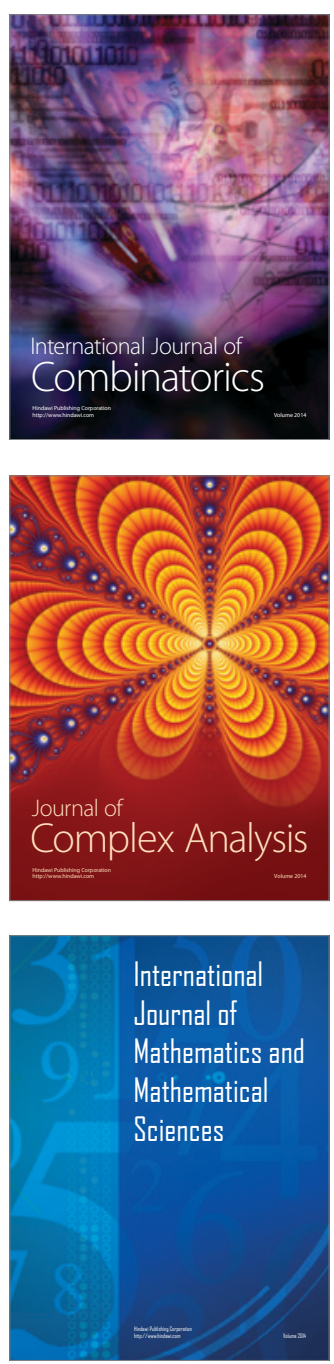
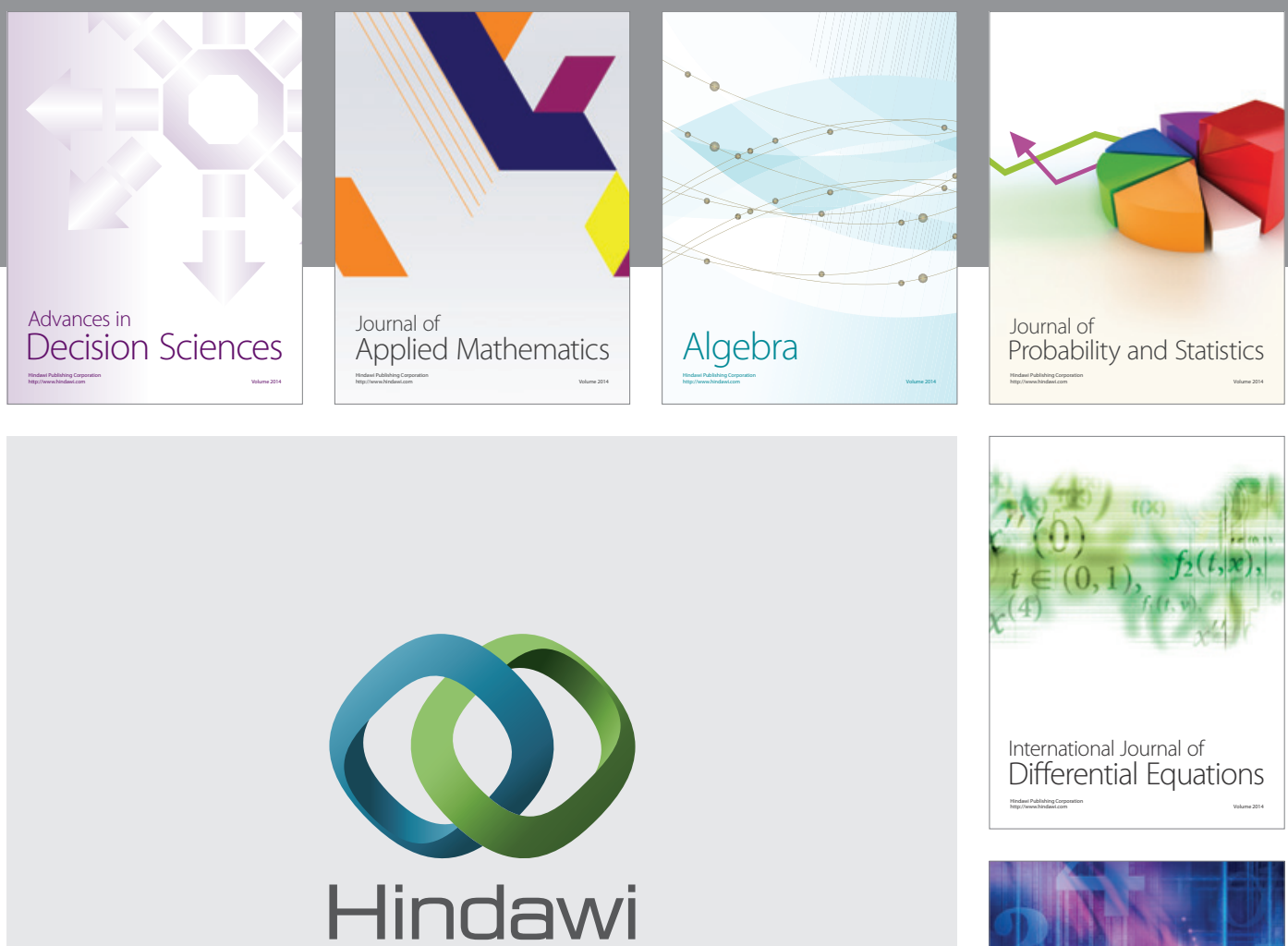

Submit your manuscripts at http://www.hindawi.com
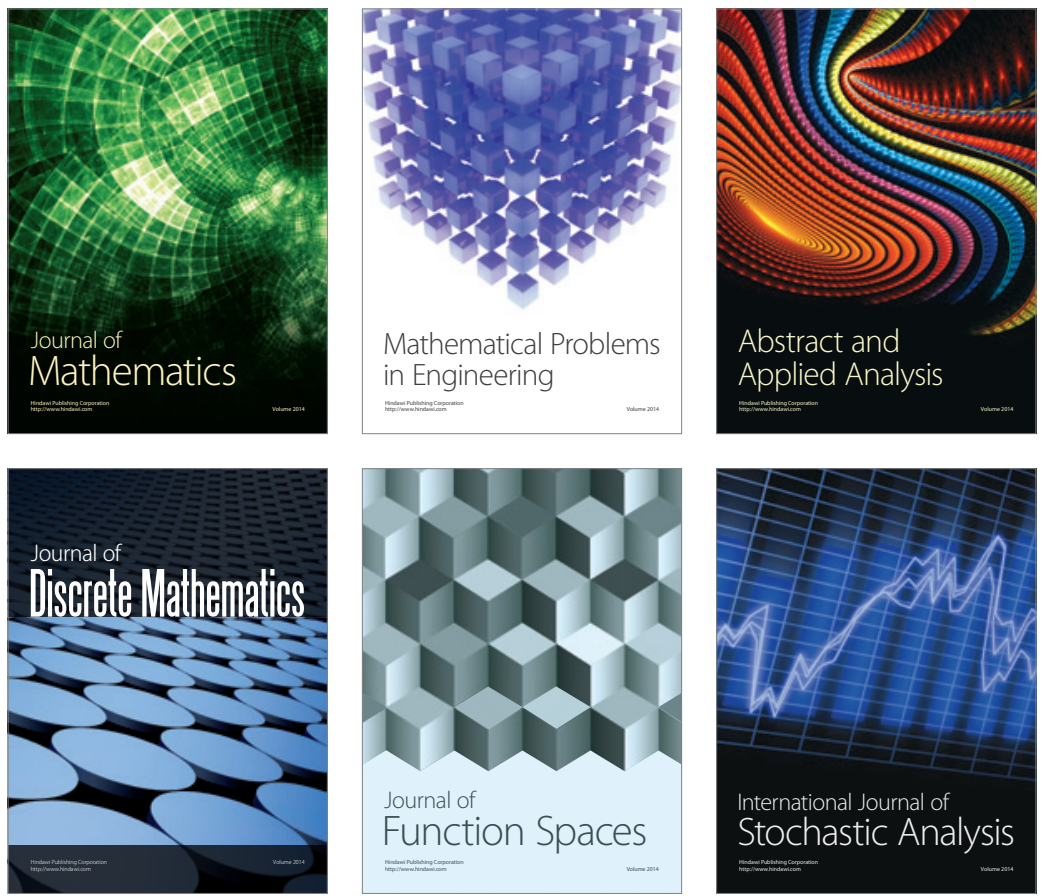

Journal of

Function Spaces

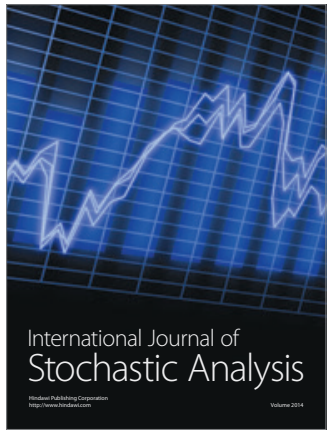

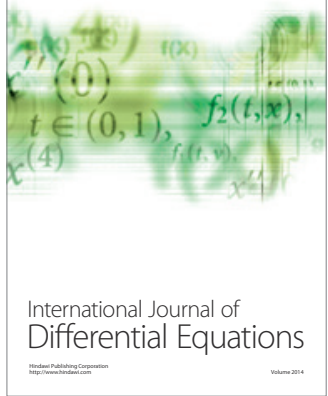
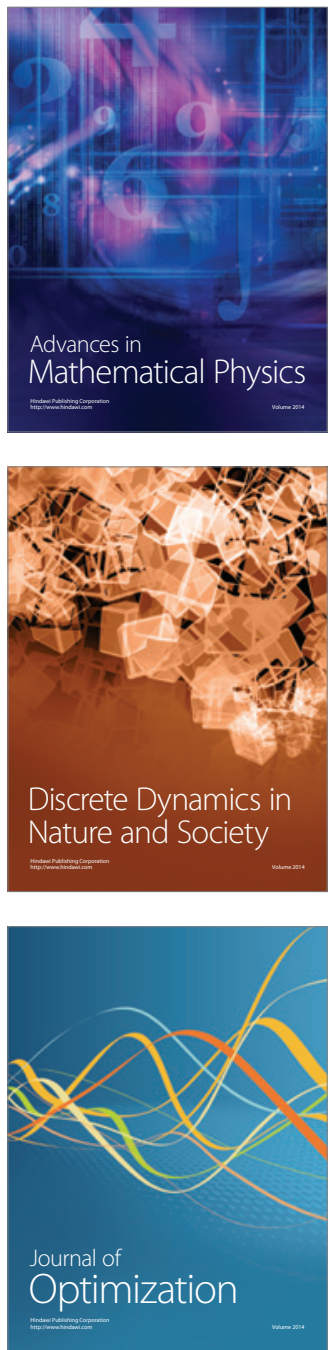